\title{
Inguinal hernia repair using 3D printed surgical instruments in the cadaveric model: A feasibility study
}

\author{
Mitchell George" ${ }^{* 1}$, Harvey Hawes ${ }^{1}$, Kevin Aroom ${ }^{1}$, Brijesh S. Gill ${ }^{1}$, and Joseph Love ${ }^{1}$ \\ ${ }^{1}$ University of Texas Medical School at Houston, USA
}

\begin{abstract}
Background: 3D printing is an additive manufacturing process allowing the creation of solid objects directly from computer aided design (CAD). Design and fabrication of 3D printed surgical instruments has previously been performed with results indicating relevance to real surgical procedures. This study expands on previous work and investigates the feasibility of performing a cadaveric inguinal hernia repair using 3D printed surgical instruments.
\end{abstract}

Methods: A CAD software package was used to design a general surgical set including hemostats, needle driver, scalpel handle, retractors and forceps. The digital models were manufactured on a 3D printer and used to perform a standard inguinal hernia repair on a human cadaver.

Results: An acceptable inguinal hernia repair including mesh placement was successfully performed on a human cadaver using standard surgical techniques.

Conclusions: General surgical procedures such as an inguinal hernia repair are feasible using $3 \mathrm{D}$ printed surgical instruments.

\section{Introduction}

The practice of surgery in the operating theater requires a spectrum of surgical instruments. Limited by storage space, availability, proximity to a supplier or cost, certain surgical centers cannot stock all of these instruments in house. On demand manufacturing of surgical instruments or components needed for surgical procedures would expand the capabilities of these centers. 3D printing is a manufacturing process with the potential to bridge this gap thanks to its high precision and relatively short build time [1]. Selective laser sintering (SLS), the type of 3D printing used in this study, manufactures parts by sintering plastic powder with a scanning laser in a layered manner [2]. As one layer of the part is finished, a roller spreads a fresh layer of powder over the build area and the laser sinters the next layer, as seen in Figure 1. The powder in the build area acts as a supporting structure for any overhanging features. Once the build is complete, the build area is raised and the part cake is taken to a breakout station for removal of excess powder. Once the excess powder and parts are separated, the powder can be reused in another build.

Original investigations applying 3D printing in the medical and surgical field included use as implants [3], construction of clinical anatomic models [4] and construction of tissue matrices [5]. 3D printing already has an established role within oral-maxillofacial surgery. Numerous reports in the literature exist where 3D printing is utilized for surgical planning and production of implants or implant guides for maxillofacial surgery [6-10]. Within the field of trauma surgery, the feasibility of functional 3D printed surgical instruments has been proven by performing a simulated laparotomy on a cut suit model [11]. Other studies have investigated the use of 3D printing in preoperative planning for orthopedic surgery [12]. Rankin et al proved the financial and structural viability of simple instruments like an Army-Navy retractor [13]. Wong et al examined the concept of printing on demand surgical instruments from digital libraries in a resource limited environment like a space station [14]. However, the technical performance of functional 3D printed surgical instruments in the operating theater on humans remains unproven. For this reason the authors propose to investigate the performance of $3 \mathrm{D}$ printed surgical instruments in surgical procedures such as an indirect inguinal hernia repair. This study builds off of previous work designing and fabricating $3 \mathrm{D}$ printed surgical instruments [15]. In the current study, the authors use computer aided design software and selective laser sintering to build a general surgical set and perform an inguinal hernia repair on a human male cadaver.

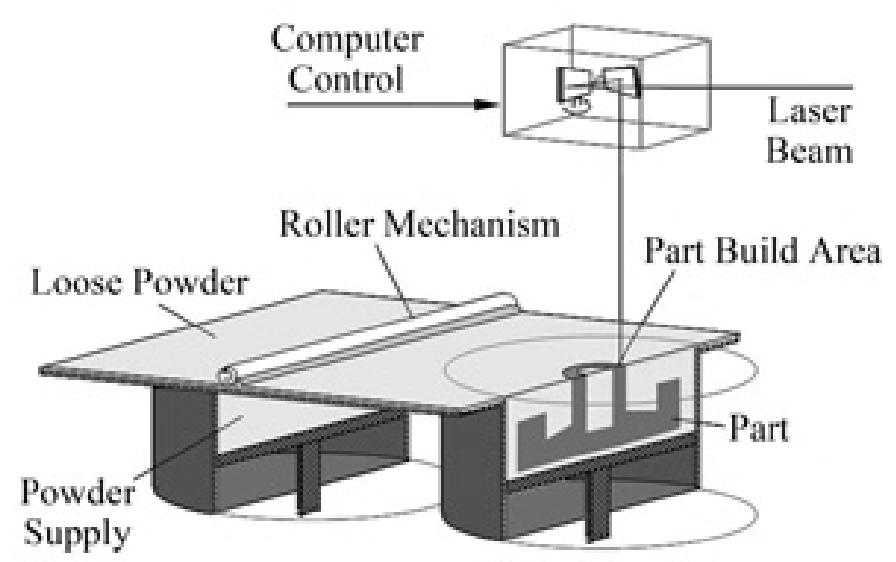

Figure 1. An SLS machine.

Correspondence to: Mitchell George, University of Texas Medical School at Houston, USA

Key words: hernia repair, $3 D$ printing, surgical instruments

Received: March 03, 2017; Accepted: March 27, 2017; Published: March 29, 2017 


\section{Materials and methods}

The design, fabrication and optimization of the general surgical set used for this evaluation has been described previously [15]. Briefly, the Computer Aided Design (CAD) package SolidWorks was used to develop digital models of the surgical instruments (Dassault Systemes SolidWorks Corp., Waltham MA). Material property limitations of the DuraForm EX plastic (3D Systems, Rock Hill SC) as compared to the standard instrument material of stainless steel necessitated instrument design modifications. Multiple iterations of needle drivers, scalpel handles and hemostats were previously performed based on surgeon feedback during simulated and cadaveric surgery. Digital models were printed using the SLS Sinterstation HiQ (3D Systems, Herts, UK) using DuraForm EX Plastic. The instruments used in the surgery were manufactured in 1 build cycle in the Sinterstation using DuraForm EX plastic powder. The build envelope of the Sinterstation is W15 x D13 x H18 inches. The instruments were oriented such that build height was minimized to decrease build time. Build height for the general surgical set was 3.1 inches and build time was 8 hours 20 minutes. Post processing to prepare the instruments for surgery included breaking the parts out of a part cake and using a combination of bead blasting and compressed air to remove excess build powder. The instruments were not sterilized because they were to be used in human cadavers. For this study the contents of the general surgical set include 1 hemostat, 1 needle driver, 1 scalpel handle, 2 forceps, one self-retaining retractor and 2 Army Navy retractors. Photographs of the instruments used in the procedure are shown in Figure 2.

To prove the functionality of the instruments, an open Lichtenstein inguinal hernia repair was performed on a male human cadaver with no obvious scarring or deformities of the groin and pelvis area. On exam there was no inguinal hernia present bilaterally. It was impossible to know if a small hernia was present on either side, however as discussed in the next paragraph there was indeed a small indirect inguinal hernia of the right side, the chosen side of the operation. Non-3D printed items used in the surgery were a \#10 scalpel blade, suture, vessel loop and mesh, seen in Figure 3.

\section{Results}

The hernia repair operation took 35 minutes, from initial skin incision to skin closure. The cadaver was placed in the supine position, and the right groin was chosen according to surgeon preference. The scalpel handle with the attached \#10 scalpel blade was used to incise the skin and subcutaneous tissue. The surgical set did not contain scissors, so the scalpel was used again to incise the external oblique without issue, from the external ring laterally $5 \mathrm{~cm}$. The fit of the blade into the handle design was snug and allowed adequate pressure exerted to cleanly divide tissue. The blade was easily removed from the handle to facilitate replacement if needed. Exposure of the surgical field was achieved by retracting the superior and inferior external oblique aponeurosis using Army Navy retractors and retracting skin and subcutaneous fat with the self-retaining retractor. Maintaining exposure using these retractors was adequate to proceed with the operation. Dissection of the external oblique off contents of the inguinal canal was aided by the hemostat and blunt dissection. The cord structures and hernia sac were then encircled first with an Army-Navy retractor. Progression to this point is sequentially shown in Figure 4.

A vessel loop was then used to encircle cord structures and hernia sac in standard fashion. The cremaster muscle was divided longitudinally then dissected off the rest of the cord structures using the forceps as seen in Figure 5. Forceps tips were fine enough to gently
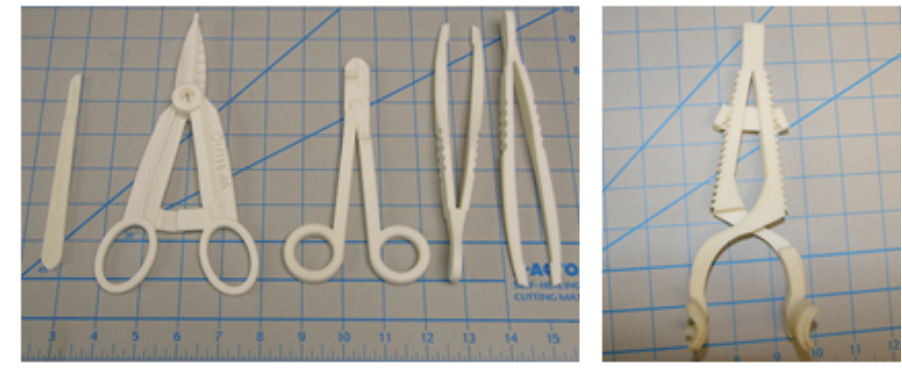

Figure 2. General surgical set including (from left to right) scalpel handle, hemostat, needle driver, forceps and self-retaining retractor. Army-Navy retractors shown elsewhere.

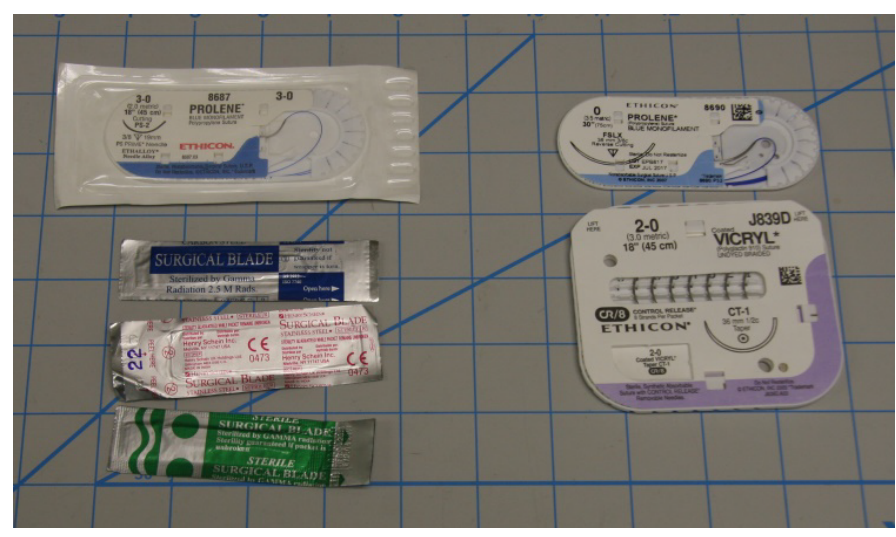

Figure 3. Non-3D printed items used in the procedure.
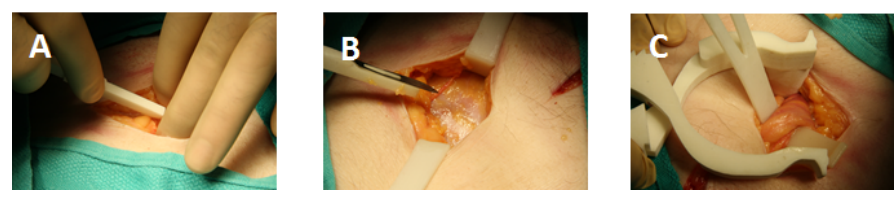

Figure 4. Incision through skin and sub-cutaneous fat, (A).Exposure of external oblique fascia, (B). Encircling cord structures and hernia sac with Army-Navy, (C).

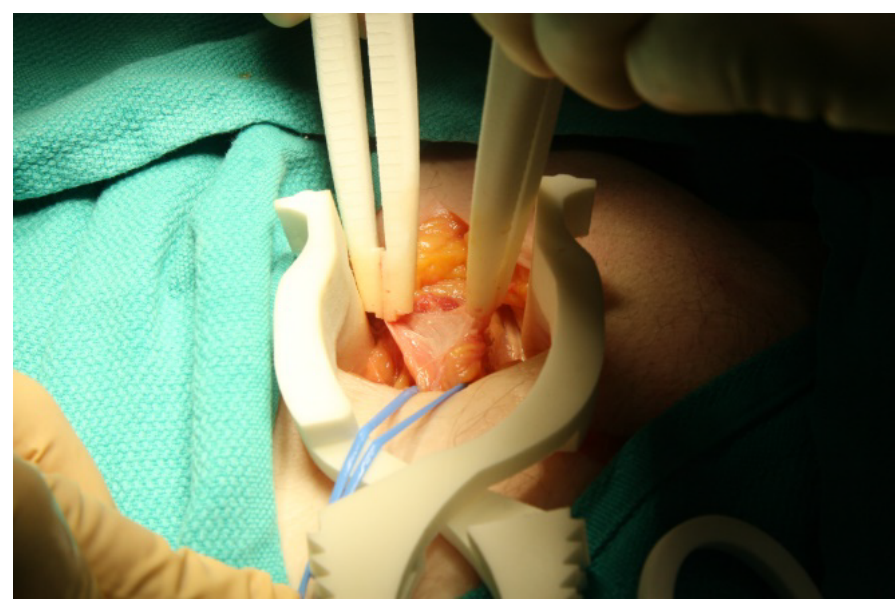

Figure 5. Spermatic cord and hernia sac is dissected out using forceps. Exposure allowed by self-retaining retractor.

dissect thin tissue veils sequentially off the cord structures and indeed, a small hernia sac was visualized.

The hemostats and forceps were used to gently dissect the sac off the cord structures. The vas deferens was identified and care was taken not to damage any other surrounding structures. The dissected sac was grasped by the hemostat, its distal portion was tied off with a 2-0 silk 
as seen in Figure 6. The tips of the hemostat had sufficient strength to grasp and lift the hernia sac for suture ligation.

The hernia sac was then excised using the scalpel. The light weight mesh was then opened and placed in the field. While driving a 3-0 Prolene suture through the pubic tubercle aponeurosis to sew the mesh in place, there was a moderate amount of slippage of the needle through the jaws of the driver as force was applied. The needle was re-grasped twice to finish passage through the aponeurosis. The mesh was then sewn into place through the inguinal ligament and around the spermatic cord using the needle driver, forceps and 3-0 Prolene suture. Driving the suture needle through these softer tissues proceeded without issue. Another technical problem with instrument functionality was crossing of the forceps tips while grasping an object that exerts forces obliquely to the grasping surfaces. Figure 7 shows this issue occurring with the forceps grasping the edge of the incised external oblique. This issue did not occur when grasped tissue planes were normal to the forceps.

Mesh placement was completed without tension and without wrinkling of the mesh. The self-retaining retractor and vessel loop were then removed. Exposure of the external oblique was facilitated by the Army-Navy retractor. The needle driver, 2-0 Vicryl suture and forceps were used to close the external oblique fascia, as seen in Figure 8.

Skin closure was done with remaining 3-0 Prolene suture as standard 3-0 monocryl suture was unavailable. Final skin closure, while not aesthetically pleasing, was performed without slippage of the needle in the needle driver. Approximation of skin edges was sufficient, as seen in Figure 9. At the end of the procedure the needle count was correct. All instruments were cleaned and inspected for cracks or signs of wear, which there was none.

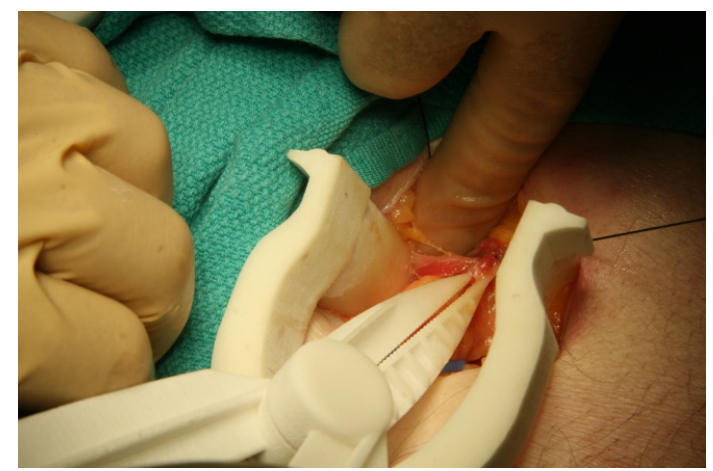

Figure 6. Suture ligation of the hernia sac using silk ties. Exposure facilitated by the selfretaining retractor.

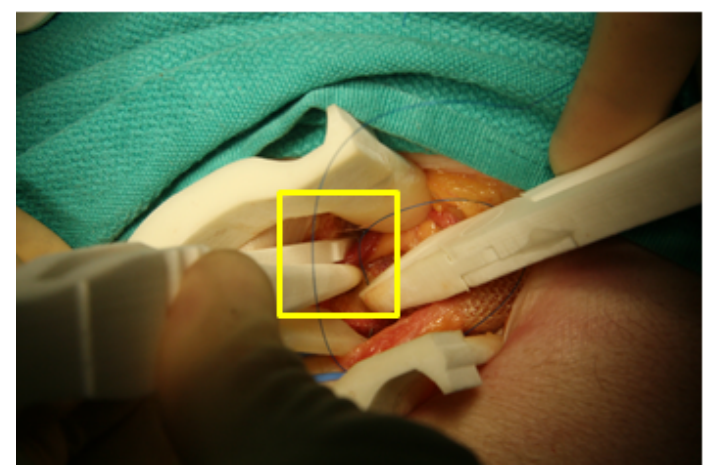

Figure 7. Forceps tip crossing while grasping external oblique aponeurosis to assist in mesh placement, highlighted in box.

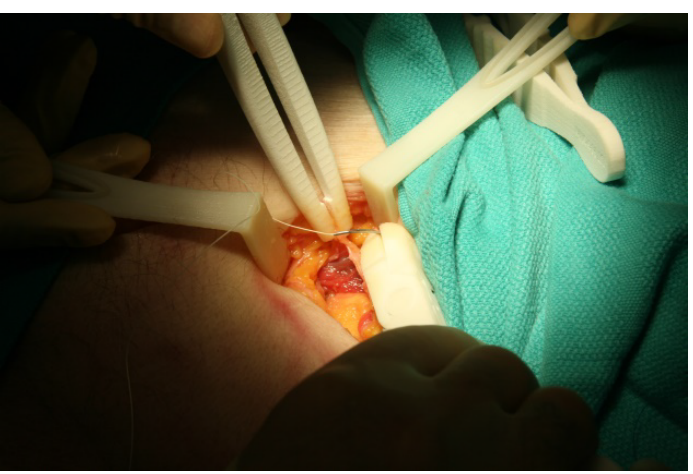

Figure 8. Closure of external oblique aponeurosis.

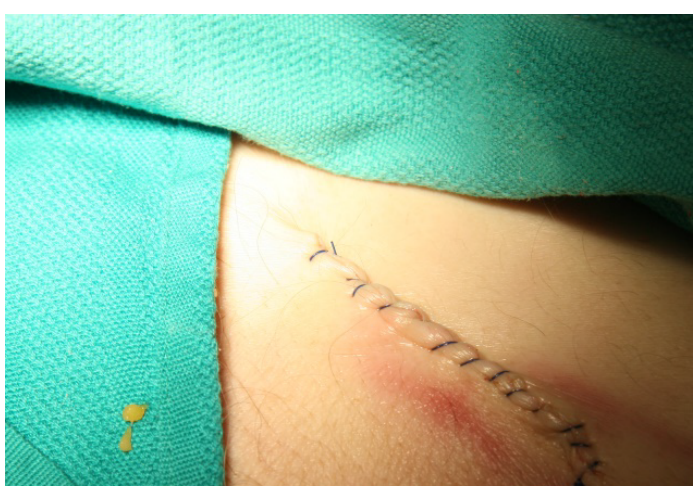

Figure 9. Final skin closure in a non-standard running fashion.

The operation was performed entirely in the cadaver laboratory. There was a single attending surgeon present who performed the operation with 2 surgery residents and 2 medical students also present assisting or taking photographs.

\section{Discussion}

To our knowledge, this is the first report of a complete general surgical operation conducted with $3 \mathrm{D}$ printed surgical instruments on a human. Overall the instruments performed sufficiently well to complete the operation. Feedback from the operating surgeon included slippage of the needle when driving through fibrous tissue like pubic tubercle aponeurosis, and crossing of forceps tips when grasping tissue at oblique angles. Future work to correct these issues include adding metallic inserts into the face of the grasping surfaces of the needle driver and altering the profile of the forceps to increase off-axis instrument stiffness.

The advantages of functional 3D printed surgical instruments are enormous. Time constraints on design and instrument production are minimal while physician customization allowed is limitless. The speed of iteration allows next day improvements to the core design of surgical instruments. Future potential applications include installment of $3 \mathrm{D}$ printers where surgical equipment is not readily available, such as forward operating surgical hospitals in combat zones, spacecraft or other resource constrained environments. These environments would be supported by on site 3D printers and have open access to digital libraries of medical and non-medical parts. These parts could be printed on demand, sterilized, used in the field and potentially recycled to build new parts. Additionally, each printable substrate could have unique sterilization properties necessitating evaluation and consideration to printing the instruments sterile in their applied environment. 
Currently, there are a number of surgical components that are not applicable to the $3 \mathrm{D}$ printing process. These include suture needles, suture and scalpel blades as seen in this study. Fabricating scissor blades was previously attempted with poor results. As the sophistication and technical ability of $3 \mathrm{D}$ printers increases, it is feasible to think that these components will be included in a 3D printed general surgical set.

The instruments used in this operation were designed on CAD software from the ground up in a matter of days. Their iterative design process and improvement is detailed previously. This study allowed for further iterative changes in the general surgical set to address instrument deficiencies. There remains much room for improvement in their design, such as improved suture needle grip of the needle drivers and increased rigidity of the forceps. Using the 3D printing manufacturing process and $\mathrm{CAD}$, these changes can be made to the original digital models and new iterations of the general surgical set can be produced in a single day.

\section{Conclusions}

In this study the authors prove that it is feasible to perform an inguinal hernia repair with a general surgical set designed by surgeons and manufactured on a 3D printer. All instruments needed to complete the operation were manufactured in one round of $3 \mathrm{D}$ printing except for a scalpel blade, vessel loop, mesh and suture. Limitations of the $3 \mathrm{D}$ printed general surgical set were primarily related to strength of the material. For example, while driving suture through the periosteum of the pubic tubercle there was some slippage of the needle requiring the needle to be re-grasped. However, the procedure was finished in 35 minutes without the aid of any standard stainless steel instruments.

\section{References}

1. Hull CWA (1986) Apparatus for production of three-dimensional objects by stereolithography. UVP, Inc. (San Gabriel, CA): United States.

2. Beaman JJA (1991) Selective laser sintering with assisted powder handling. 1991, Board of Regents, The University of Texas System (Austin, TX): United States.
3. Berry E, Brown JM, Connell M, Craven CM, Efford ND, et al. (1997) Preliminary experience with medical applications of rapid prototyping by selective laser sintering. Med Eng Phys 19: 90-96. [Crossref]

4. Mankovich NJ, Cheeseman AM, Stoker NG (1990) The display of three-dimensional anatomy with stereolithographic models. J Digit Imaging 3: 200-203. [Crossref]

5. Ripamonti U (1992) Calvarial reconstruction in baboons with porous hydroxyapatite. $J$ Craniofac Surg 3: 149-159. [Crossref]

6. Flugge TV, Nelson K, Schmelzeisen R, Metzger MC, et al. (2013) Three-dimensiona plotting and printing of an implant drilling guide: simplifying guided implant surgery. J Oral Maxillofac Surg 71: 1340-1346. [Crossref]

7. Shaheen E, Sun Y, Jacobs R, Politis C (2017) Three-dimensional printed final occlusal splint for orthognathic surgery: design and validation. Int J Oral Maxillofac Surg 46: 67-71. [Crossref]

8. Aboul-Hosn Centenero S, Hernandez-Alfaro F (2012) 3D planning in orthognathic surgery: $\mathrm{CAD} / \mathrm{CAM}$ surgical splints and prediction of the soft and hard tissues results our experience in 16 cases. J Craniomaxillofac Surg 40: 162-168. [Crossref]

9. Lill W, Solar P, Ulm C, Watzek G, Blahout R, et al. (1992) Reproducibility of three-dimensional CT-assisted model production in the maxillofacial area. Br J Oral Maxillofac Surg 30: 233-236. [Crossref]

10. Silva DN, Gerhardt de Oliveira M, Meurer E, Meurer MI, Lopes da Silva JV, et al. (2008) Dimensional error in selective laser sintering and 3D-printing of models for craniomaxillary anatomy reconstruction. J Craniomaxillofac Surg 36: 443-449. [Crossref]

11. Shayne Kondor M (2013) On Demand Additive Manufacturing of a Basic Surgical Kit. J Med Dev 7: 030916.

12. Hurson C, Tansey A, O'Donnchadha B, Nicholson P, Rice J, et al. (2007) Rapid prototyping in the assessment, classification and preoperative planning of acetabular fractures. Injury 38: 1158-1162. [Crossref]

13. Rankin TM, Giovinco NA, Cucher DJ, Watts G, Hurwitz B, et al. (2014) Threedimensional printing surgical instruments: are we there yet?. J Surg Res 189: 193-197. [Crossref]

14. Wong JY, Pfahnl AC (2016) 3D Printed Surgical Instruments Evaluated by a Simulated Crew of a Mars Mission. Aerosp Med Hum Perform 87: 806-810. [Crossref]

15. George M, Aroom KR, Hawes HG, Gill BS, Love J (2017) 3D Printed Surgical Instruments: The Design and Fabrication Process. World J Surg 41: 314-319. [Crossref]

Copyright: $\odot 2017$ George M. This is an open-access article distributed under the terms of the Creative Commons Attribution License, which permits unrestricted use, distribution, and reproduction in any medium, provided the original author and source are credited. 